\title{
Pilocytic astrocytomas of the optic nerve and their relation to pilocytic astrocytomas elsewhere in the central nervous system
}

\author{
Gerald F Reis ${ }^{1}$, Michele M Bloomer ${ }^{2}$, Arie Perry ${ }^{1}$, Joanna J Phillips ${ }^{1,3}$, James P Grenert ${ }^{1}$, \\ Anthony N Karnezis ${ }^{1}$ and Tarik Tihan ${ }^{1}$ \\ ${ }^{1}$ Neuropathology Unit, Department of Anatomic Pathology, UCSF School of Medicine, San Francisco, \\ CA, USA; ${ }^{2}$ Department of Ophthalmology, UCSF School of Medicine, San Francisco, CA, USA and \\ ${ }^{3}$ Department of Neurological Surgery, UCSF School of Medicine, San Francisco, CA, USA
}

\begin{abstract}
Pilocytic astrocytoma is a low-grade glioma that affects mostly children and young adults and can occur anywhere in the central nervous system. Pilocytic astrocytoma of the optic nerve is an equally indolent subtype that is occasionally associated with neurofibromatosis type 1 . In earlier studies, this subtype was considered within the larger category of 'optic pathway glioma,' which included infiltrating astrocytomas and other hypothalamic tumors. However, there have been suggestions that gliomas in the optic nerve, and especially pilocytic astrocytoma of the optic nerve, are biologically different from tumors within the hypothalamus and other parts of the optic tract. Furthermore, the recent discovery of BRAF duplication and fusion with the KIAA1549 gene is reported to be more typical for posterior fossa tumors, and the rate of this aberration is not well known in pilocytic astrocytoma of the optic nerve. To determine the distinction of pilocytic astrocytoma of the optic nerve from pilocytic astrocytoma of the posterior fossa and to investigate the prevalence of BRAF aberrations, we reviewed the clinicopathological and molecular features of all such patients in our institution. Our study demonstrates that BRAF duplication is more frequent in posterior fossa tumors compared with pilocytic astrocytoma of the optic nerve $(P=0.011)$. However, the rates of phospho-MAPK1 and CDKN2A expression were high in both pilocytic astrocytoma of the optic nerve and posterior fossa pilocytic astrocytoma, suggesting that the MAPK pathway is active in these tumors. Our study supports the notion that BRAF duplication is more typical of posterior fossa pilocytic astrocytoma and that molecular alterations other than KIAA1549 fusion may underlie MAPK pathway activation in pilocytic astrocytoma of the optic nerve. Modern Pathology (2013) 26, 1279-1287; doi:10.1038/modpathol.2013.79; published online 24 May 2013
\end{abstract}

Keywords: optic glioma; pediatrics; pilocytic astrocytoma

Pilocytic astrocytoma is one of the most common central nervous system glial neoplasms seen in the pediatric population. These tumors occur most frequently in patients between the ages of 0 and 14 years with a predilection for the infratentorial region., ${ }^{1,2}$ Pilocytic astrocytoma is classified as a World Health Organization (WHO) grade I neoplasm given its excellent prognosis and long-term progressionfree survival even after subtotal tumor resection., ${ }^{2,3}$ However, approximately a fifth of the patients recur

Correspondence: Dr T Tihan, MD, PhD, Neuropathology Unit, Department of Anatomic Pathology, UCSF School of Medicine, Room M551, 505 Parnassus Avenue, San Francisco, CA 941430102, USA.

E-mail: tarik.tihan@ucsf.edu

Received 10 December 2012; revised 24 February 2013; accepted 27 February 2013; published online 24 May 2013 after resection ${ }^{4}$ and undergo adjuvant therapy, which carries significant toxicity and morbidity.

Optic glioma is a nonspecific clinical term that includes pilocytic astrocytoma of the optic nerve, infiltrating gliomas, and hypothalamic tumors. While the majority of optic gliomas are in the pilocytic category, the inclusion of other entities can render high diversity to the group, in terms of pathological features and prognosis. In addition, tumors in different locations along the optic pathway may also have different characteristics. Thus, studies directed specifically at pilocytic astrocytoma of the optic nerve are needed to provide better insight on the biology and behavior of these tumors in comparison to tumors located elsewhere along the optic pathway and also in relation to tumors with overlapping histological features located elsewhere in the central nervous system. 
As with all pilocytic astrocytomas, the majority of pilocytic astrocytomas of the optic nerve are sporadic, although an increased frequency is seen in neurofibromatosis type 1 patients. ${ }^{5}$ Sporadic- and neurofibromatosis type 1-associated pilocytic astrocytomas typically behave in an indolent manner, with some tumors showing spontaneous regression after subtotal resection or in some cases even without any surgery. ${ }^{6}$ On the other hand, hypothalamic tumors include pilocytic astrocytomas with more aggressive behavior, such as the pilomyxoid astrocytoma, that have higher rates of recurrence or cerebral spinal fluid dissemination. ${ }^{7}$ These findings suggest that pilocytic astrocytoma represents a more heterogeneous group with varying histologic patterns and biologic behaviors. This heterogeneity also includes biological differences due to site, histological variants, and molecular features, requiring accurate characterization of variables to identify objective predictive and prognostic markers.

A growing body of evidence suggests that the duplication/rearrangement of $B R A F$ at $7 q 34$ and generation of the KIAA1549:BRAF fusion product is the most common molecular alteration in sporadic pilocytic astrocytoma, occurring at highest frequency in tumors of the posterior fossa. ${ }^{8-13}$ The biologic significance of $B R A F$ duplication lies in the activation of the MAPK pathway, which can drive tumor proliferation. ${ }^{9}$ In addition to KIAA1549:BRAF fusion product, other molecular alterations have been reported in pilocytic astrocytoma, including other $B R A F$ fusion products, ${ }^{14,15}$ rare $B R A F^{V 600 E}$ mutations, ${ }^{16} \quad B R A F$ insertions, ${ }^{17}$ and KRAS mutations. ${ }^{18}$ This list further highlights the critical importance of the MAPK pathway in these tumors. The remaining question is the type and degree of influence that each genetic alteration exerts on this pathway and whether specific genetic alterations can be recognized in relation to tumor location and behavior.

While the clinical features of pilocytic astrocytoma of the optic nerve have been fairly well characterized, the relation of these tumors to pilocytic astrocytoma located elsewhere in the central nervous systemand especially those in the posterior fossa-is less established, as prior studies on $B R A F$ duplication and activation of the MAPK pathway included only rare examples from the optic nerve, except for a recent study showing a high rate of $B R A F$ duplication. ${ }^{19}$ Thus, we evaluated the clinicopathological and molecular features of pilocytic astrocytoma of the optic nerve to determine whether these tumors can be distinguished from pilocytic astrocytoma located elsewhere in the central nervous system.

\section{Materials and methods}

\section{Patients}

A database search was performed for all pilocytic astrocytoma of the optic nerve diagnosed and treated at the University of California Medical Center between 1954 and 2010. In addition, existing data were utilized from a recent multi-institutional study of 116 intracranial pilocytic astrocytomas for comparison. ${ }^{20}$ Clinical information such as gender, tumor location, type of surgery, and extent of resection was obtained from a review of medical records. Inclusion criteria required that tumors involved only the optic nerve or optic chiasm and that slides were available for review to confirm the diagnosis. Consultation cases were excluded. Recurrence was defined as the emergence of a new radiologically defined lesion or lesions that required treatment. All pathology specimens were reviewed by three of the authors (MB, TT, and GR) to confirm the diagnosis of pilocytic astrocytoma according to the criteria by the WHO. Appropriate permission for the study was obtained from the University of California San Francisco Committee for Human Research (CHR 10-01252).

\section{Tissue Microarray}

Of 19 cases identified, 13 had adequate neoplastic tissue for immunohistochemical and molecular analysis. Two representative 2-mm cores were taken from designated areas of each available tumor block, and samples from autopsy specimens of unremarkable brains were used as non-neoplastic controls.

\section{Immunohistochemistry}

Immunohistochemistry was performed using an automated immunohistochemical staining processor at the University of California San Francisco Immunohistochemistry Laboratory and Brain Tissue Research Center (Leica Byosystems). After antigen retrieval with $\mathrm{pH} 6.0$ citrate buffer, we performed immunohistochemistry for EMA, GFAP, synaptophysin, Neu-N, OLIG2, phospho-MAPK1 (p-MAPK1), CDKN2A, and Ki-67. For cytoplasmic markers, the following antibodies and dilutions were used: EMA (Dako; 1:120), GFAP (Dako; 1:5000), synaptophysin (SPH; Dako; 1:150), p-MAPK1 (Zymed; $50 \mu \mathrm{g} / \mathrm{ml}$ ), and CDKN2A (Mtm Lab provided the kit). For nuclear markers, the following antibodies and dilutions were used: Neu-N (Chemicon; 1:2000), OLIG2 (Dako; 1:250), and Ki-67 (Dako; 1:1000). Previously prepared tissue microarrays from our intracranial pilocytic astrocytoma cohort were obtained for comparative analysis of CDKN2A. Tissue microarrays for WHO grade II and III oligodendrogliomas $(N=68)$, as well as grade IV glioblastomas $(N=60)$ were also used. Scoring for cytoplasmic antibodies was carried out using a semiquantitative method described previously. ${ }^{20}$ In brief, we determined the ratio of tumor cells staining positive to those staining negative for each antibody without consideration of staining intensity. Cases were semiquantitatively scored as follows (\% staining in 
tumor cells; cytoplasmic staining): (0) no staining (negative); (1) staining in $<25 \%$; (2) staining between 25 and $50 \%$; (3) staining between 50 and $75 \%$; and (4) staining in $>75 \%$. Nuclear staining was scored by counting approximately 1000 cells (with a focus on areas of maximal staining) and calculating a labeling index (number of positive cells divided by the total number of cells, expressed as a percentage).

\section{Fluorescence In Situ Hybridization Analysis}

We assessed BRAF duplication by fluorescence in situ hybridization (FISH) performed on tissue microarrays as described previously. ${ }^{20-22}$ For $B R A F$, we used the RP11-355D18 (KIAA) and RP4-726N20 $(B R A F)$ probes labeled with fluoroisothiocyanate (green) and rhodamine (red), respectively. Nonneoplastic samples were used as controls. At least 100 non-overlapping nuclei were assessed for the presence of fused signals, with a positive result defined by at least $25 \%$ of tumor cells displaying both evidence of copy number gain (at least three red and/or green signals) in combination with at least one yellow fusion signal due to overlapping red and green signals. For CDKN2A copy number alterations, we utilized the LSI CDKN2A (9p21) orange and CEP9 green Spectrum probes (Abbott Laboratories). Hybridizations were interpreted as described previously ${ }^{22,23}$ and were considered deleted regardless of whether the pattern suggested hemizygous or homozygous losses.

\section{$B R A F^{V 600 E}$ Mutation Analysis}

The presence of the $B R A F^{V 600 E}$ point mutation was assessed as described previously. ${ }^{24}$ In brief, genomic DNA was isolated from formalin-fixed paraffinembedded tissue using proteinase $\mathrm{K}(3 \mathrm{mg} / \mathrm{ml})$ digestion solution ( $50 \mathrm{mM}$ Tris, $1 \mathrm{mM}$ EDTA, $\mathrm{pH}$ 8.0, $1 \%$ Tween-20). ${ }^{25}$ Samples were incubated at $55^{\circ} \mathrm{C}$ overnight (approximately $12 \mathrm{~h}$ ), and the supernatant was transferred into a newly labeled microcentrifuge tube after brief centrifugation. Proteinase $\mathrm{K}$ was inactivated by incubating the samples at $95{ }^{\circ} \mathrm{C}$ for $10 \mathrm{~min}$. The DNA was diluted to a working concentration of $50 \mathrm{ng} / \mathrm{ml}$ before amplification. Primers were designed to amplify a $250 \mathrm{bp}$ region of exon 15 in the BRAF gene: forward, $5^{\prime}$-CTC TTCATAATGCTTGCTCTGATAGG-3'; reverse, $5^{\prime}$-TAG TAACTCAGCAGCATCTCAGG-3' (Integrated DNA Technologies, Coralville, IA, USA). Fluorescent hybridization probes were designed to detect the $B R A F^{V 600 E}$ wild-type sequence: sensor, 5' ${ }^{\prime}$-AGCTAC AGTGAAATCTCGATGGAG-fluoroscein-3'; anchor, 5'-LCRed640-GGTCCCATCAGTTTGAACAGTTGTC TGGA-phosphate- $3^{\prime}$, with the sensor probe spanning nucleotide position 1799 (IdahoTechnologies, Salt Lake City, UT, USA). The reaction underwent 45 cycles of rapid PCR consisting of denaturation at
$94{ }^{\circ} \mathrm{C}$ for $1 \mathrm{~s}$, annealing at $55^{\circ} \mathrm{C}$ for $20 \mathrm{~s}$, and extension at $72{ }^{\circ} \mathrm{C}$ for $10 \mathrm{~s}$. Transition rates were $20^{\circ} \mathrm{C} / \mathrm{s}$ from denaturation to annealing, $20^{\circ} \mathrm{C} / \mathrm{s}$ annealing to extension, and $20^{\circ} \mathrm{C} / \mathrm{s}$ extension to denaturation. Post-amplification fluorescent melting curve analysis was performed by gradual heating of the samples at a rate of $0.1^{\circ} \mathrm{C} / \mathrm{s}$ from 45 to $95^{\circ} \mathrm{C}$. Fluorescent melting peaks were determined by plotting of the negative derivative of fluorescence with respect to temperature.

\section{Statistics}

For descriptive data of variables with a normal distribution, mean and standard deviation were used. For variables for which a normal distribution was not assumed, median and range were used. Comparisons among population characteristics were made using the $\chi^{2}$ and Fisher's exact tests, and comparisons among nominal variables were made using the Student's $t$-test. Results with a $P$-value $<0.05$ were accepted as statistically significant.

\section{Results}

The study cohort consisted of 14 girls and 5 boys $(N=19$; Table 1). Most patients presented with proptosis (65\%) and vision loss (40\%). Additional signs included extraocular movement defects, esotropia, blurring of the optic disc, optic atrophy, and pupillary light defects with dilated and fixed pupil, as well as absent reaction to light. One patient had a history of grand mal seizures. A total of eight patients $(42 \%)$ underwent a subtotal resection, whereas four patients $(21 \%)$ underwent a gross total resection; the extent of resection could not be determined for the remaining 7 (37\%). Subsequent to initial surgery, three patients received adjuvant therapy (one patient received radiation, one patient received chemotherapy, and one patient received both radiation and chemotherapy). The patient who received radiation alone was a 10-month-old neurofibromatosis type 1 baby boy with an optic nerve pilocytic astrocytoma involving the bilateral optic nerves and optic chiasm. The baby developed irritability and fussiness with marked proptosis of the left globe following surgery. The radiotherapy was felt to yield relief to the symptoms. The baby subsequently underwent an uncomplicated left eye and optic nerve enucleation but died 8 months later because of unknown causes (an autopsy was not performed). The patient who received chemoradiation was a 4-year-old girl with progressive proptosis of the right eye. She developed radiation-induced orbital edema and optic neuritis and underwent hyperbaric oxygen therapy following radiation. The chemotherapy was complicated by gastrointestinal symptoms, limiting medication dose. On follow-up imaging 7 months after the initiation of therapy, there was apparent stabilization in lesion size. 
Table 1 Pertinent clinical features of 19 patients with pilocytic astrocytoma of the optic nerve

\begin{tabular}{|c|c|}
\hline Patient characteristics & No. of cases (percentage) \\
\hline \multicolumn{2}{|l|}{ Age } \\
\hline Median & 120 months \\
\hline Mean & 118 months \\
\hline \multicolumn{2}{|l|}{ Gender } \\
\hline Male & $5(26 \%)$ \\
\hline Female & $14(74 \%)$ \\
\hline \multicolumn{2}{|l|}{ Location } \\
\hline Optic nerve & $16(84 \%)$ \\
\hline Optic chiasm & $1(5 \%)$ \\
\hline Both & $2(11 \%)$ \\
\hline \multicolumn{2}{|c|}{ Lateralization (cases affecting only the optic nerve) } \\
\hline Right & $7(44 \%)$ \\
\hline Left & $8(50 \%)$ \\
\hline Both & $1(6 \%)$ \\
\hline NF-1 & $4(21 \%)$ \\
\hline \multicolumn{2}{|l|}{ Surgery } \\
\hline Subtotal resection & $8(42 \%)$ \\
\hline Gross total resection & $4(21 \%)$ \\
\hline Unknown & $7(37 \%)$ \\
\hline Recurrence & $4(21 \%)$ \\
\hline \multicolumn{2}{|l|}{ Adjunct therapy } \\
\hline Chemotherapy & $1(5 \%)$ \\
\hline Radiotherapy & $1(5 \%)$ \\
\hline Both & $1(5 \%)$ \\
\hline \multicolumn{2}{|l|}{ Outcome } \\
\hline Died of disease & $1(5 \%)$ \\
\hline Alive with tumor & $1(5 \%)$ \\
\hline Lost to follow-up & $17(89 \%)$ \\
\hline
\end{tabular}

Because of prominent proptosis and disfigurement, however, the patient underwent a tumor debulking procedure approximately 19 months later. On follow-up imaging 10 months later, there was evidence of tumor progression, and the patient underwent an excision. She was alive and without evidence of disease progression 63 months later. The patient who received chemotherapy alone was a 3-year-old boy with a chiasmatic pilocytic astrocytoma. Although therapeutic records were not available for review, the patient was alive and without evidence of recurrence at the end of the follow-up period (216 months).

The mean follow-up period for the entire cohort was 39 months (range 0-216 months). At the end of the follow-up period, 10 patients (53\%) were alive and 1 patient $(5 \%)$ had died; the remaining 8 patients $(42 \%)$ were lost to follow-up. Four patients (21\%) experienced disease recurrence (range 5-108 months) and underwent a second surgical procedure: two patients underwent an enucleation, one patient underwent a gross total resection, and one patient underwent a subtotal resection. Of the four patients who had a second surgical procedure, two subsequently presented with disease recurrence and underwent a third surgical procedure: the status of 1 patient was after enucleation 6 years earlier, and the other status was after subtotal resection 24 months earlier. These two patients underwent excision and subtotal resection, respectively, and showed no evidence of recurrence 13 and 8 months later (last follow-up visit). The mean progression-free survival for the cohort was 33 months.

In comparison to the 116 intracranial pilocytic astrocytoma cohort previously reported by our group, ${ }^{20}$ pilocytic astrocytoma of the optic nerve showed a significant higher number of affected girls $(P=0.03)$ but no difference in terms of outcome $(P=0.86)$, extent of resection $(P=0.7)$, recurrence $(P=0.37)$, neurofibromatosis type 1 status $(P=0.92)$, age of diagnosis $(P=0.11)$, chemotherapy $(P=0.31)$, or radiotherapy $(P=0.24)$.

\section{Histologic Features}

In general, pilocytic astrocytoma of the optic nerve elicited meningothelial hyperplasia of the orbital connective tissue in $30 \%$ of the cases (Figures 1a and 2a). The underlying optic nerve showed moderate to marked hypercellularity with distortion of the normal nerve columnar pattern or complete loss of septa. Clusters of neoplastic cells demonstrated fibrillar cytoplasm. The nuclei were often enlarged and pale, with one or more tiny nucleoli (Figure 1b). Rosenthal fibers were present in 15 $(79 \%)$ of cases, 6 of which $(40 \%)$ also contained eosinophilic granular bodies (Figure 1c). Loose microcystic areas with variable mucinous/myxoid stroma were seen in 33\% of the cases (Figure 1d). Mitotic figures were inconspicuous.

\section{Immunohistochemical Features}

We evaluated select cases using antibodies to markers of glial and neuronal differentiation (see Materials and methods; Figure 2 and Table 2). The neoplastic cells were typically strongly positive for GFAP (Figure 2b) and negative for Neu-N (Figure 2c). In $85 \%$ of the cases, GFAP demonstrated category 4 staining. Most cases $(77 \%)$ had extensive OLIG2 positivity (Figure $2 \mathrm{~d}$ ), $62 \%$ of which were scored as category 3 or 4 staining. Synaptophysin positivity was present in $46 \%$ of the cases (Figure 2e), typically in the same areas that were GFAP positive and within long astrocyte-like processes rather than more typical neuronal morphology. For one-half of the cases, Ki-67 was entirely negative. For the other half, only rare cells stained with Ki-67, yielding labeling indices of $<1 \%$. This immunohistochemical pattern was similar to that observed for intracranial pilocytic astrocytoma.

We analyzed activation of the MAPK pathway with antibodies to p-MAPK1 and CDKN2A (Figure 2 and Table 2). Approximately $81 \%$ of the cases showed staining for p-MAPK1 (Figure 2f), with 
a
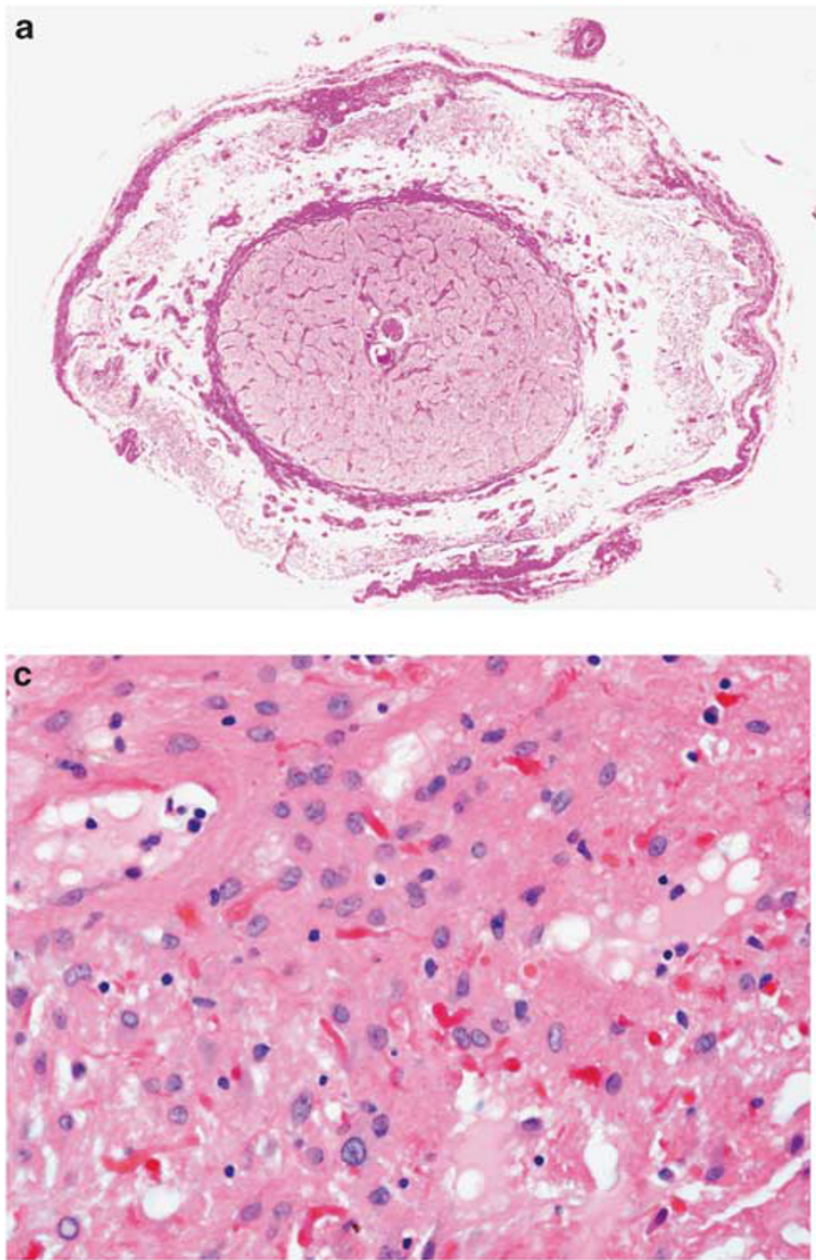
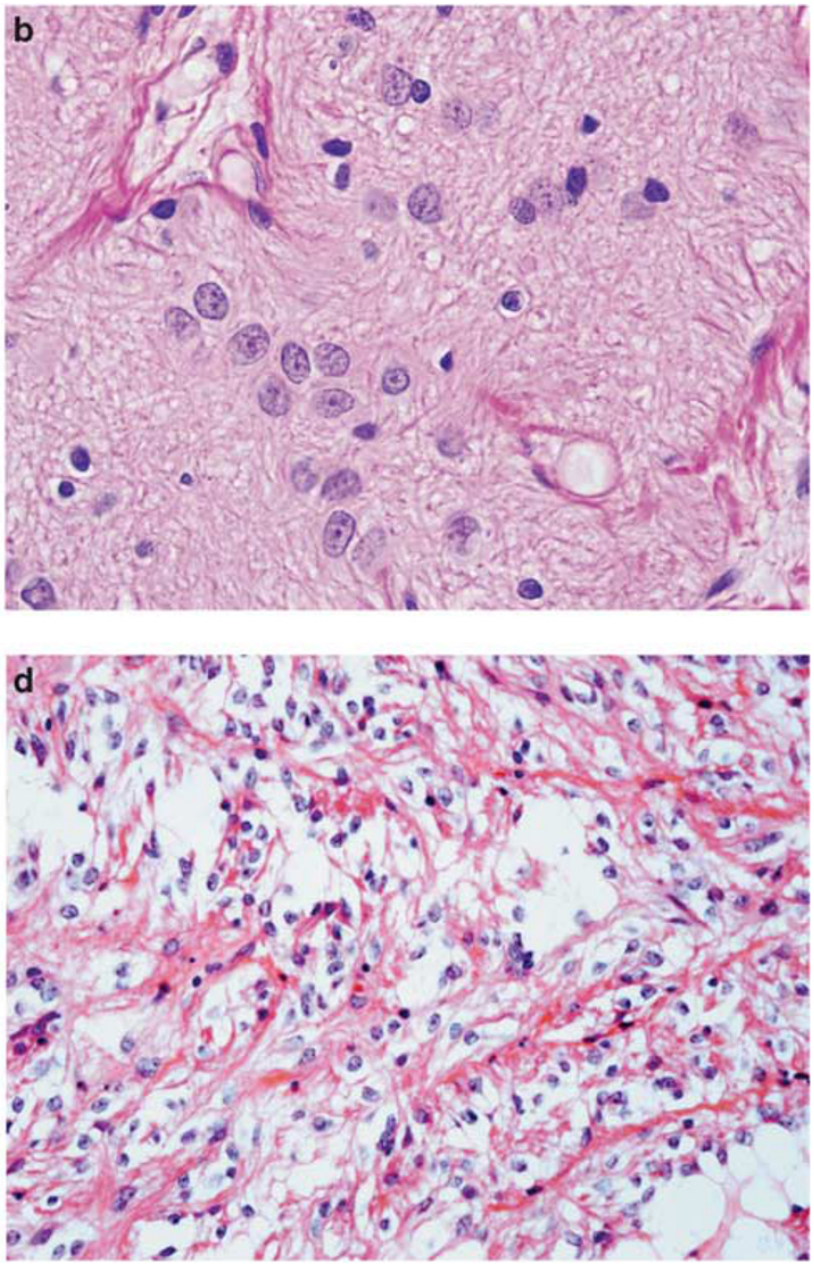

Figure 1 Histologic features of pilocytic astrocytoma of the optic nerve. Pilocytic astrocytoma of the optic nerve elicited meningothelial hyperplasia (a). The neoplastic cells frequently exhibited enlarged, pale nuclei with numerous small nucleoli (b). The neoplastic cells were interspersed within a fibrillary and fibrous connective tissue background with numerous Rosenthal fibers (c). Loose areas containing mucinous material were seen in some of the cases $(\mathbf{d})$.

$62 \%$ of the cases displaying category 3 or 4 staining. No staining was identified in $19 \%$ of the cases. This pattern was significantly different $(P<0.0001)$ from that seen for intracranial pilocytic astrocytoma, with a higher proportion of neoplastic cells staining for p-MAPK1 in pilocytic astrocytoma of the optic nerve. All pilocytic astrocytomas of the optic nerve were positive for CDKN2A (Figure $2 \mathrm{~g}$ ), and $62 \%$ of the cases displayed category 3 or 4 staining. While intracranial pilocytic astrocytoma was also positive for CDKN2A, it demonstrated category 3 or 4 staining only in $5 \%$ of cases, which was significantly different from pilocytic astrocytoma of the optic nerve $(P<0.01)$.

To evaluate CDKN2A expression in higher-grade gliomas in comparison to pilocytic astrocytoma of the optic nerve, we performed CDKN2A staining in a group of oligodendrogliomas (WHO grades II and III) and glioblastomas (WHO grade IV). Of the 68 oligodendrogliomas tested, $32(47 \%)$ showed CDKN2A staining, although only $11.8 \%$ was category 2 or higher (Table 2). In glioblastomas, 57 of 60 cases showed CDKN2A staining, but only $33 \%$ were category 2 or higher. A significantly higher percentage of neoplastic cells in pilocytic astrocytoma of the optic nerve was positive for CDKN2A as compared with oligodendroglioma or glioblastoma (oligodendroglioma, $P<0.001$; GBM, $P<0.01$ ).

\section{FISH Findings}

Of the 13 cases tested, 5 were negative (39\%; Figure 2h), 3 were positive (23\%; Figure 2i), and 5 were indeterminate $(39 \%)$. The latter represented mostly older cases ( $>30$ years), where it is well recognized that rates of success for FISH are considerably diminished. Compared with pilocytic astrocytoma of the posterior fossa, ${ }^{20}$ the observed frequency of BRAF duplication in pilocytic astrocytoma of the optic nerve was significantly less $(P=0.011$; Fischer's exact test). We also used FISH 

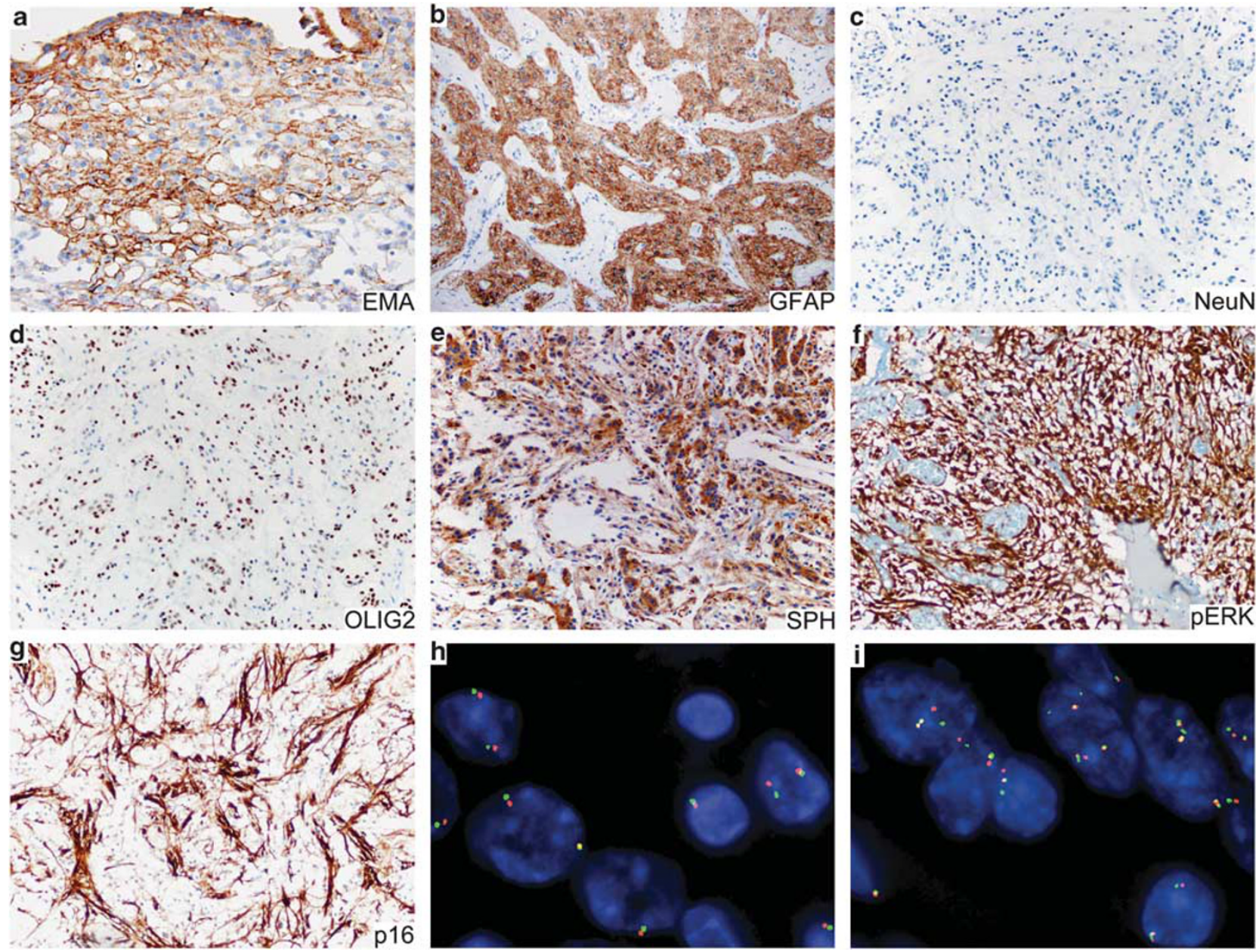

Figure 2 Immunohistochemical and molecular features of pilocytic astrocytoma of the optic nerve. The epithelial membrane antigen (EMA) highlighted foci of meningothelial hyperplasia (a). The neoplastic cells were positive for glial fibrillary acidic protein (GFAP) (b) and negative for neuronal nuclei (Neu-N) (c). Numerous neoplastic cells were positive for OLIG2 (d), and positivity for synaptophysin was also observed (e). Strong staining for phospho-mitogen-activated protein kinase 1 (p-MAPK1) (f) and CDKN2A (g) were a feature of pilocytic astrocytoma of the optic nerve. KIAA1549:BRAF fusion was negative in 39\% of cases (h) and positive in $23 \%$ of cases (i).

to evaluate for CDKN2A deletions in pilocytic astrocytoma of the optic nerve and found no evidence of CDKN2A alterations. Of the 13 cases tested, $11(85 \%)$ were negative and $2(15 \%)$ were indeterminate.

\section{$B R A F^{V 600 E}$ Findings}

We tested pilocytic astrocytoma of the optic nerve for $B R A F^{V 600 E}$ and found 1 positive case $(8 \%)$ of 13 cases tested. Seven cases (54\%) were negative, and the remaining $5(38 \%)$ were indeterminate. Interestingly, the one positive $B R A F^{V 600 E}$ case also showed KIAA1549:BRAF fusion by FISH.

\section{Discussion}

Our study evaluated the clinicopathological and molecular features of pilocytic astrocytoma of the optic nerve to determine whether these tumors can be distinguished from pilocytic astrocytoma located elsewhere in the central nervous system. The data demonstrate that pilocytic astrocytoma of the optic nerve has similar clinicopathological features to posterior fossa pilocytic astrocytoma, including age of diagnosis, neurofibromatosis type 1 status, extent of resection, adjuvant therapy, tumor recurrence, and outcome, although the small size for our data set $(N=19)$ is a shortcoming in our study. The shorter follow-up period for pilocytic astrocytoma of the optic nerve constitutes another shortcoming, but this is often suggested to be related to the tumor's favorable biologic behavior. Most patients without neurofibromatosis type 1 do not seek further treatment, especially after a gross total resection of the neoplasm. Pilocytic astrocytoma of the optic nerve has been reported to regress spontaneously subsequent to subtotal resection or even without surgery. ${ }^{6}$ For these reasons, the term optic glioma should be avoided when referring to these tumors. Several patients in our cohort underwent an enucleation or 
Table 2 Immunohistochemical results: (A) cytoplasmic antibodies, (B) nuclear antibodies, and (C) CDKN2A

\begin{tabular}{|c|c|c|c|c|c|c|c|}
\hline \multirow{2}{*}{ Antigen } & & \multirow{2}{*}{\multicolumn{2}{|c|}{$\mathrm{N}$}} & \multicolumn{4}{|c|}{ Staining } \\
\hline & & & & 0 & 2 & 3 & 4 \\
\hline \multicolumn{8}{|l|}{ (A) } \\
\hline GFAP & & & 13 & 0 & 0 & 1 & 11 \\
\hline Synapt & $y \sin$ & & 13 & 7 & 0 & 0 & 0 \\
\hline p-MAP & & & 13 & 2 & 0 & 3 & 6 \\
\hline Antigen & $\mathrm{N}$ & \multicolumn{2}{|c|}{ Mean LI (\%) } & \multicolumn{2}{|c|}{ Median LI (\%) } & \multicolumn{2}{|c|}{ Range (\%) } \\
\hline \multicolumn{8}{|l|}{ (B) } \\
\hline Neu-N & 13 & & 0 & & 0 & & N/A \\
\hline OLIG2 & 13 & & 55 & & 63 & & $5-90$ \\
\hline \multirow[t]{3}{*}{ Ki-67 } & 13 & & $<1$ & & $<1$ & & N/A \\
\hline & & \multirow[b]{2}{*}{$\mathrm{N}$} & \multicolumn{5}{|c|}{ Staining } \\
\hline & & & $O(\%)$ & $1(\%)$ & $2(\%)$ & $3(\%)$ & $4(\%)$ \\
\hline \multicolumn{8}{|l|}{ (C) } \\
\hline \multicolumn{2}{|c|}{$\begin{array}{l}\text { Pilocytic of } \\
\text { optic nerve }\end{array}$} & 13 & $0(0)$ & $1(8)$ & $4(31)$ & $3(23)$ & $5(38)$ \\
\hline \multicolumn{2}{|c|}{$\begin{array}{l}\text { Pilocytic of } \\
\text { posterior fossa }\end{array}$} & 116 & $8(7)$ & 41 (35) & 46 (39) & $17(15)$ & $4(3)$ \\
\hline \multicolumn{2}{|c|}{$\begin{array}{l}\text { Oligodendro- } \\
\text { glioma }\end{array}$} & 68 & $36(53)$ & $24(36)$ & $4(6)$ & $3(4)$ & $1(2)$ \\
\hline \multicolumn{2}{|c|}{ Glioblastoma } & 60 & $2(4)$ & $38(63)$ & $6(10)$ & $4(7)$ & $10(16)$ \\
\hline
\end{tabular}

gross total resection and required no further treatment. On the other hand, our longest followup periods correlated with subtotal resection cases requiring multiple repeat resections. This suggests that the risk of recurrence is still associated with the extent of surgical resection, which is not surprising and is consistent with the biology reported for pilocytic astrocytoma in all locations. Although the number of patients undergoing adjuvant therapy was limited in our study, radiation and chemotherapy did not appear to provide significant benefit and were associated with a few complications.

The histological and immunohistochemical features of pilocytic astrocytoma of the optic nerve were similar to those of intracranial pilocytic astrocytoma. Both pilocytic astrocytoma of the optic nerve and intracranial pilocytic astrocytoma show expansive growth, Rosenthal fibers, eosinophilic granular bodies, biphasic appearance, and rare mitotic figures. We did not encounter cases with pilomyxoid or anaplastic features. Like intracranial pilocytic astrocytoma, the immunohistochemical features of pilocytic astrocytoma of the optic nerve included positivity for GFAP, OLIG2, and synaptophysin, while being largely negative for Neu-N. We also tested for alterations in the MAPK pathway, as these have been proposed to drive tumor proliferation in low-grade gliomas. ${ }^{26,27}$ Our analysis of downstream factors in the MAPK pathway demonstrated positivity for p-MAPK1 and CDKN2A in both pilocytic astrocytoma of the optic nerve and intracranial pilocytic astrocytoma. However, pilo- cytic astrocytoma of the optic nerve had a significantly higher proportion of cells positive for p-MAPK1 and CDKN2A (p-MAPK1, $P<0.0001$; CDKN2A, $P<0.01)$. When compared with highergrade gliomas, CDKN2A positivity in pilocytic astrocytoma of the optic nerve was also significantly higher (oligodendroglioma, $P<0.001$; GBM, $P<0.01$ ). While these findings are not likely due to poor tissue quality, artifactual changes cannot be entirely excluded given some of our samples were nearly 50 years old.

One of the upstream activators of the MAPK pathway is the oncogene $B R A F$, and many studies have documented $B R A F$ alterations in pilocytic astrocytoma. $^{8,9,18,20}$ Our molecular analysis of BRAF in pilocytic astrocytoma of the optic nerve showed KIAA1549:BRAF fusion and BRAF V600E point mutation, which further supports the presence of $B R A F$ alterations in pilocytic astrocytoma. However, the rate of $B R A F$ fusion in pilocytic astrocytoma of the optic nerve was significantly lower than for posterior fossa pilocytic astrocytoma (optic nerve, $38 \%$; posterior fossa, $84 \% ; P=0.011$, Fischer's exact test). This is consistent with previous studies suggesting a higher frequency of the KIAA1549:BRAF fusion in posterior fossa pilocytic astrocytoma. ${ }^{18,20}$ Of note, our findings on BRAF differ from a recently reported pilocytic astrocytoma of the optic nerve cohort, ${ }^{19}$ where the frequency of KIAA1549:BRAF fusion $(73 \%)$ was similar to that of posterior fossa pilocytic astrocytoma. The reason for this difference is not clear, and the two cohorts of pilocytic astrocytomas of the optic nerve showed no significant difference in terms of gender, neurofibromatosis type 1 status, median age at diagnosis, and outcome. However, the presence of a group of pilocytic astrocytoma of the optic nerve tumors without $B R A F$ alterations suggests that molecules other than $B R A F$ may be associated with pilocytic astrocytoma of the optic nerve. Interestingly, one of our cases tested positive for both $B R A F$ duplication and $B R A F^{V 600 E}$ mutation. The presence of both alterations has been recently reported for a low-grade astrocytoma. ${ }^{28}$ As there is only a single such case, the effect of both alterations on tumor behavior, while curious, is not clear.

Even though the presence of $B R A F$ alterations was significantly less frequent in pilocytic astrocytoma of the optic nerve, these tumors showed strong staining for p-MAPK1 and CDKN2A. The presence of other less common activating alterations in $B R A F$ is one possible explanation for our findings. Among the $B R A F$ fusion products reported in pilocytic astrocytoma, ${ }^{28}$ we only tested for KIAA1549:BRAF. This raises the possibility that other alterations in $B R A F$ may drive the activation of $\mathrm{p}-\mathrm{MAPK} 1$ and CDKN2A in pilocytic astrocytoma of the optic nerve. Alternatively, alterations in negative regulatory factors of the MAPK pathway, such as the Sprouty family of proteins, Raf kinase inhibitor protein, impedes mitogenic signal propagation, and the 


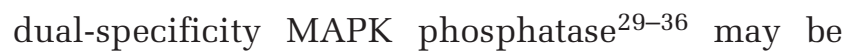
found in pilocytic astrocytoma of the optic nerve. Studies are currently under way to test for these possibilities.

The BRAF/MEK/MAPK1 pathway has been implicated in oncogene-induced senescence, a biological process in which tumors induced to grow by an oncogenic alteration ultimately undergo permanent growth arrest. ${ }^{37-39}$ The presence of BRAF alterations and MAPK1 activation in pilocytic astrocytoma of the optic nerve raises the possibility of an active oncogene-induced senescence in these tumors. Activation of oncogene-induced senescence may provide a potential explanation for the long periods of dormancy and/or spontaneous regression reported in pilocytic astrocytoma. ${ }^{40}$ Because this particular pathway can induce senescence through activation of CDKN2A, ${ }^{41}$ positivity for CDKN2A may be a surrogate for the activation of oncogene-induced senescence in pilocytic astrocytoma. Indeed, a worse clinical outcome has been reported for pilocytic astrocytoma lacking CDKN2A expression. ${ }^{42}$ The extent of follow-up in our study permitted only limited evaluation of survival and correlation with the molecular features of pilocytic astrocytoma of the optic nerve, and future studies will be needed to address the relationship between CDKN2A activation and clinical behavior in these tumors. Nevertheless, the presence of an active MAPK pathway and indirect evidence of increased oncogene-induced senescence in pilocytic astrocytoma of the optic nerve can explain their indolent behavior. Of course, one caveat of treating tumors with an activated oncogene-induced senescence may be introduction of additional mutations in checkpoints such as CDKN2A or TP53, which allow them to escape senescence and become aggressive. ${ }^{43}$ Indeed, low-grade gliomas treated with radiotherapy have been reported to recur as anaplastic tumors. ${ }^{44}$

In summary, the elucidation of the MAPK pathway as the centerpiece of alterations in pilocytic astrocytoma provides a better understanding of these tumors, which become chronic diseases with a protracted and arduous course. Our data along with an increasing body of evidence in the literature suggest that indolent behavior in pilocytic astrocytoma of the optic nerve is associated with oncogeneinduced senescence initiated by the activation of the MAPK pathway. Therapy regimens that can abrogate oncogene-induced senescence through inactivation of molecules such as CDKN2A or TP53 are more likely to be associated with aggressive outcome and will need to be avoided. This evidence also confirms the historical wisdom of earlier studies that warn us about overtreating such tumors. ${ }^{45}$

\section{Acknowledgements}

We thank Hansel Ihn and Dr Yunn-Yi Chen for the help in generating the tissue microarray of pilocytic astrocytoma of the optic nerve, Marvin Mungcal for assistance with histologic sections, Ann Halili for aid with immunohistochemistry, and Roxanne Marshall for the help with the FISH assays. We thank the University of California San Francisco molecular laboratory staff for assistance with the $B R A F^{V 600 E}$ assay. This research was supported by the Residents' Teaching and Research Endowments from the University of California San Francisco Department of Pathology.

\section{Disclosure/conflict of interest}

The authors declare no conflict of interest.

\section{References}

1 Rosemberg S, Fujiwara D. Epidemiology of pediatric tumors of the nervous system according to the WHO 2000 classification: a report of 1,195 cases from a single institution. Childs Nerv Syst 2005;21:940-944.

2 Scheithauer BW, Hawkins C, Tihan T, et al. Pilocytic astrocytoma, In: Louis DN, Ohgaki H, Wiestler OD, Cavenee WK (eds). WHO Classification of Tumours of the Central Nervous System. IARC Press: Lyon, France, 2007, pp 14-21.

3 Ohgaki H, Kleihues P. Population-based studies on incidence, survival rates, and genetic alterations in astrocytic and oligodendroglial gliomas. J Neuropathol Exp Neurol 2005;64:479-489.

4 Dirven CM, Mooij JJ, Molenaar WM. Cerebellar pilocytic astrocytoma: a treatment protocol based upon analysis of 73 cases and a review of the literature. Childs Nerv Syst 1997;13:17-23.

5 Listernick R, Charrow J, Greenwald MJ, et al. Optic gliomas in children with neurofibromatosis type 1. J Pediatr 1989;114:788-792.

6 Parsa CF, Hoyt CS, Lesser RL, et al. Spontaneous regression of optic gliomas: thirteen cases documented by serial neuroimaging. Arch Ophthalmol 2001;119: $516-529$

7 Tihan T, Fisher PG, Kepner JL, et al. Pediatric astrocytomas with monomorphous pilomyxoid features and a less favorable outcome. J Neuropathol Exp Neurol 1999;58:1061-1068.

8 Jones DT, Kocialkowski S, Liu L, et al. Tandem duplication producing a novel oncogenic BRAF fusion gene defines the majority of pilocytic astrocytomas. Cancer Res 2008;68:8673-8677.

9 Bar EE, Lin A, Tihan T, et al. Frequent gains at chromosome 7q34 involving BRAF in pilocytic astrocytoma. J Neuropathol Exp Neurol 2008;67:878-887.

10 Jacob K, Albrecht S, Sollier C, et al. Duplication of 7q34 is specific to juvenile pilocytic astrocytomas and a hallmark of cerebellar and optic pathway tumours. Br J Cancer 2009;101:722-733.

11 Pfister S, Janzarik WG, Remke M, et al. BRAF gene duplication constitutes a mechanism of MAPK pathway activation in low-grade astrocytomas. J Clin Invest 2008;118:1739-1749.

12 Sievert AJ, Jackson EM, Gai X, et al. Duplication of $7 q 34$ in pediatric low-grade astrocytomas detected by high-density single-nucleotide polymorphism-based 
genotype arrays results in a novel BRAF fusion gene. Brain Pathol 2009;19:449-458.

$13 \mathrm{Yu}$ J, Deshmukh H, Gutmann RJ, et al. Alterations of BRAF and HIPK2 loci predominate in sporadic pilocytic astrocytoma. Neurology 2009;73:1526-1531.

14 Jones DT, Kocialkowski S, Liu L, et al. Oncogenic RAF1 rearrangement and a novel BRAF mutation as alternatives to KIAA1549:BRAF fusion in activating the MAPK pathway in pilocytic astrocytoma. Oncogene 2009;28:2119-2123.

15 Cin H, Meyer C, Herr R, et al. Oncogenic FAM131BBRAF fusion resulting from 7q34 deletion comprises an alternative mechanism of MAPK pathway activation in pilocytic astrocytoma. Acta Neuropathol 2011;121:763-774.

16 Schindler G, Capper D, Meyer J, et al. Analysis of BRAF V600E mutation in 1,320 nervous system tumors reveals high mutation frequencies in pleomorphic xanthoastrocytoma, ganglioglioma and extra-cerebellar pilocytic astrocytoma. Acta Neuropathol 2011;121: 397-405.

17 Eisenhardt AE, Olbrich H, Roring M, et al. Functional characterization of a BRAF insertion mutant associated with pilocytic astrocytoma. Int J Cancer 2011;129: 2297-2303.

18 Forshew T, Tatevossian RG, Lawson AR, et al. Activation of the ERK/MAPK pathway: a signature genetic defect in posterior fossa pilocytic astrocytomas. J Pathol 2009;218:172-181.

19 Rodriguez FJ, Ligon AH, Horkayne-Szakaly I, et al. BRAF duplications and MAPK pathway activation are frequent in gliomas of the optic nerve proper. J Neuropathol Exp Neurol 2012;71:789-794.

20 Tihan T, Ersen A, Qaddoumi I, et al. Pathologic characteristics of pediatric intracranial pilocytic astrocytomas and their impact on outcome in 3 countries: a multiinstitutional study. Am J Surg Pathol 2012;36:43-55.

21 Kim YH, Nonoguchi N, Paulus W, et al. Frequent BRAF gain in low-grade diffuse gliomas with $1 \mathrm{p} / 19 \mathrm{q}$ loss. Brain Pathol 2012;22:834-840.

22 Korshunov A, Meyer J, Capper D, et al. Combined molecular analysis of BRAF and IDH1 distinguishes pilocytic astrocytoma from diffuse astrocytoma. Acta Neuropathol 2009;118:401-405.

23 Horbinski C, Hamilton RL, Nikiforov Y, et al. Association of molecular alterations, including BRAF, with biology and outcome in pilocytic astrocytomas. Acta Neuropathol 2010;119:641-649.

24 Rowe LR, Bentz BG, Bentz JS. Detection of BRAF V600E activating mutation in papillary thyroid carcinoma using PCR with allele-specific fluorescent probe melting curve analysis. J Clin Pathol 2007;60:1211-1215.

25 Rowe LR, Bentz BG, Bentz JS. Utility of BRAF V600E mutation detection in cytologically indeterminate thyroid nodules. Cytojournal 2006;3:10.

26 Jeuken JW, Wesseling P. MAPK pathway activation through BRAF gene fusion in pilocytic astrocytomas; a novel oncogenic fusion gene with diagnostic, prognostic, and therapeutic potential. J Pathol 2010; 222:324-328.

27 Tatevossian RG, Lawson AR, Forshew T, et al. MAPK pathway activation and the origins of pediatric lowgrade astrocytomas. J Cell Physiol 2010;222:509-514.
28 Lin A, Rodriguez FJ, Karajannis MA, et al. BRAF alterations in primary glial and glioneuronal neoplasms of the central nervous system with identification of 2 novel KIAA1549:BRAF fusion variants. J Neuropathol Exp Neurol 2012;71:66-72.

29 Courtois-Cox S, Genther Williams SM, Reczek EE, et al. A negative feedback signaling network underlies oncogene-induced senescence. Cancer Cell 2006;10: 459-472.

30 Murphy T, Hori S, Sewell J, et al. Expression and functional role of negative signalling regulators in tumour development and progression. Int J Cancer 2010;127:2491-2499.

31 Matheny SA, Chen C, Kortum RL, et al. Ras regulates assembly of mitogenic signalling complexes through the effector protein IMP. Nature 2004;427:256-260.

32 Yeung K, Janosch P, McFerran B, et al. Mechanism of suppression of the Raf/MEK/extracellular signalregulated kinase pathway by the raf kinase inhibitor protein. Mol Cell Biol 2000;20:3079-3085.

33 Owens DM, Keyse SM. Differential regulation of MAP kinase signalling by dual-specificity protein phosphatases. Oncogene 2007;26:3203-3213.

34 Kim HJ, Bar-Sagi D. Modulation of signalling by Sprouty: a developing story. Nat Rev Mol Cell Biol 2004;5:441-450.

35 Boutros T, Chevet E, Metrakos P. Mitogen-activated protein (MAP) kinase/MAP kinase phosphatase regulation: roles in cell growth, death, and cancer. Pharmacol Rev 2008;60:261-310.

36 Kwak HJ, Kim YJ, Chun KR, et al. Downregulation of Spry2 by miR-21 triggers malignancy in human gliomas. Oncogene 2011;30:2433-2442.

37 Campisi J, d'Adda di Fagagna F. Cellular senescence: when bad things happen to good cells. Nat Rev Mol Cell Biol 2007;8:729-740.

38 Evan GI, d'Adda di Fagagna F. Cellular senescence: hot or what? Curr Opin Genet Dev 2009;19:25-31.

39 Cagnol S, Chambard JC. ERK and cell death: mechanisms of ERK-induced cell death-apoptosis, autophagy and senescence. FEBS J 2010;277:2-21.

40 Borit A, Richardson EP Jr. The biological and clinical behaviour of pilocytic astrocytomas of the optic pathways. Brain 1982;105:161-187.

41 Serrano M, Lin AW, McCurrach ME, et al. Oncogenic ras provokes premature cell senescence associated with accumulation of p53 and p16INK4a. Cell 1997;88: 593-602.

42 Raabe EH, Lim KS, Kim JM, et al. BRAF activation induces transformation and then senescence in human neural stem cells: a pilocytic astrocytoma model. Clin Cancer Res 2011;17:3590-3599.

43 Horbinski C, Nikiforova MN, Hagenkord JM, et al. Interplay among BRAF, p16, p53, and MIB1 in pediatric low-grade gliomas. Neuro Oncol 2012;14: 777-789.

44 Dirks PB, Jay V, Becker LE, et al. Development of anaplastic changes in low-grade astrocytomas of childhood. Neurosurgery 1994;34:68-78.

45 Burger PC, Scheithauer BW, Lee RR, et al. An interdisciplinary approach to avoid the overtreatment of patients with central nervous system lesions. Cancer 1997;80:2040-2046. 